\section{Cross-sectional and Prospective Associations of Rest-Activity Rhythms With Metabolic Markers and Type 2 Diabetes in Older Men}

Diabetes Care 2020;43:2702-2712 | https://doi.org/10.2337/dc20-0557

\section{OBJECTIVE}

Disruption of rest-activity rhythms is cross-sectionally associated with metabolic disorders, including type 2 diabetes, yet it remains unclear whether it predicts impaired glucose metabolism and homeostasis. The aim of this study is to examine the cross-sectional and prospective associations between rest-activity rhythm characteristics and glycemic measures in a cohort of older men.

\section{RESEARCH DESIGN AND METHODS}

Baseline rest-activity rhythms were derived from actigraphy with use of extended cosine model analysis. With subjects fasting, glucose, insulin, and HOMA of insulin resistance (HOMA-IR) were measured from blood at baseline and after $\sim 3.5$ years. Type 2 diabetes was defined based on self-report, medication use, and fasting glucose.

\section{RESULTS}

In the cross-sectional analysis $(n=2,450)$, lower 24-h amplitude-to-mesor ratio (i.e., mean activity-adjusted rhythm amplitude) and reduced overall rhythmicity were associated with higher fasting insulin and HOMA-IR (all $\boldsymbol{P}_{\text {trend }}<\mathbf{0 . 0 0 0 1}$ ), indicating increased insulin resistance. The odds of baseline type 2 diabetes were significantly higher among those in the lowest quartile of amplitude (Q1) (odds ratio [OR] $]_{\mathrm{Q} 1 \mathrm{vs.} \mathrm{Q4}}$ $1.63[95 \% \mathrm{Cl} 1.14,2.30])$ and late acrophase group $\left(\mathrm{OR}_{\text {late vs. normal }} 1.46\right.$ [95\% $\mathrm{Cl} 1.04$, 2.04]). In the prospective analysis $(n=861)$, multiple rest-activity characteristics predicted a two- to threefold increase in type $\mathbf{2}$ diabetes risk, including a lower amplitude ( $\mathrm{OR}_{\mathrm{Q} 1}$ vs. Q4 3.81 [95\% $\left.\left.\mathrm{Cl} 1.45,10.00\right]\right)$ and amplitude-to-mesor ratio (OR 2.79 [95\% Cl 1.10, 7.07]), reduced overall rhythmicity (OR 3.49 [95\% Cl 1.34, 9.10]), and a late acrophase (OR $2.44[1.09,5.47])$.

\section{CONCLUSIONS}

Rest-activity rhythm characteristics are associated with impaired glycemic metabolism and homeostasis and higher risk of incident type 2 diabetes.

The rest-activity cycle, which encompasses major daily activities including sleep, physical activity, and sedentary behaviors, plays an important role in metabolic health. Low levels of physical activity and prolonged sedentary behaviors are well-established risk factors for metabolic diseases (1), and sleep disturbances in the forms of short sleep, misaligned sleep timing, and irregular sleep have also been linked to metabolic dysfunction, such as obesity (2), dyslipidemia (3), metabolic syndrome (4), and type 2
Qian Xiao, ${ }^{1}$ Jingyi Qian, ${ }^{2,3}$ Daniel S. Evans, ${ }^{4}$ Susan Redline, ${ }^{5}$ Nancy E. Lane, ${ }^{6}$

Sonia Ancoli-Israel, ${ }^{7,8}$ Frank A.J.L. Scheer, ${ }^{2,3}$ and Katie Stone, ${ }^{4}$ for the Osteoporotic Fractures in Men (MrOS) Study Group
${ }^{1}$ Department of Epidemiology, Human Genetics and Environmental Health, School of Public Health, University of Texas Health Science Center at Houston, Houston, TX

${ }^{2}$ Medical Chronobiology Program, Division of Sleep and Circadian Disorders, Departments of Medicine and Neurology, Brigham and Women's Hospital, Boston, MA

${ }^{3}$ Division of Sleep Medicine, Department of Medicine, Harvard Medical School, Boston, MA

${ }^{4}$ Research Institute, California Pacific Medical Center Research Institute, San Francisco, CA

${ }^{5}$ Division of Sleep and Circadian Disorders, Brigham and Women's Hospital and Beth Israel Deaconess Medical Center, Boston, MA

${ }^{6}$ Center for Musculoskeletal Health, Medicine and Rheumatology, University of California at Davis School of Medicine, Sacramento, CA ${ }^{7}$ Departments of Psychiatry and Medicine, University of California, San Diego, La Jolla, CA ${ }^{8}$ Department of Veterans Affairs San Diego Center of Excellence for Stress and Mental Health, La Jolla, CA

Corresponding author: Qian Xiao, qian.xiao@ uth.tmc.edu

Received 18 March 2020 and accepted 15 August 2020

This article contains supplementary material online at https://doi.org/10.2337/figshare.12816059.

(C) 2020 by the American Diabetes Association. Readers may use this article as long as the work is properly cited, the use is educational and not for profit, and the work is not altered. More information is available at https://www.diabetesjournals .org/content/license. 
diabetes $(5,6)$. Although previous studies have demonstrated that physical activity, sedentary behaviors, and sleep play a strong role in metabolic health, most studies have focused on individual behaviors (e.g., physical activity, sedentary behaviors, and sleep) and failed to integrate different yet interrelated daily behaviors to capture the overall pattern of 24-h restactivity rhythms and evaluate its unique effects on metabolic health. In particular, examining the strength, regularity, and timing of the rest-activity rhythms in relation to metabolic indicators may help identify novel intervention targets for the development of preventive and therapeutic strategies to specifically improve the rhythmicity of diurnal behaviors and enhance metabolic health.

The risk for impaired glucose control and type 2 diabetes increases with age. On the other hand, aging also influences many aspects of rest-activity rhythms (7). Previous research has linked advanced age with decreases in physical activity and total sleep time and efficiency; increases in sedentary behaviors, sleep latency, and fragmentation; and shifted sleep timing including early awakening (8-12). There is also evidence suggesting that age-related disturbances in restactivity patterns, such as reduced total sleep time and efficiency and increase in sleep fragmentation, are more pronounced in men than in women $(13,14)$. Given the high prevalence of impairment in restactivity rhythms in the elderly, particularly in older men, it is important to examine its potential health effects in this population.

A few recent studies used 24-h actigraphy data to examine characteristics of 24-h rest-activity rhythms in relation to metabolic health in the older population. Almost all studies focused on rhythm regularity and fragmentation and found that reduced regularity and increased fragmentation, which suggest impaired restactivity rhythms, are associated with higher BMI, metabolic syndrome, obesity, type 2 diabetes, and dyslipidemia in older men and women $(15,16)$. Rest-activity rhythms are multidimensional, and other characteristics such as amplitude, timing, and overall shape and/or rhythmicity have been shown to be important predictors of health outcomes in the older population $(17,18)$, but their relationships with metabolic health remain unexplored. Moreover, to the best of our knowledge, there has been no study investigating the prospective relationship between restactivity rhythm characteristics and metabolic health in the older population. Given the temporal ambiguity of predictors and outcomes in cross-sectional studies, investigating the prospective relationship between rest-activity rhythm characteristics and metabolic health in older populations can contribute to a better understanding of causal relationships.

To address the aforementioned gaps in the literature, we studied both the crosssectional and prospective relationships between rest-activity rhythm patterns and metabolic health outcomes in a cohort of older men. Specifically, we hypothesized that weakened rest-activity rhythms at baseline, characterized by a lower strength of rhythmicity (i.e., amplitude, amplitude-to-mesor ratio), and average level of activity (i.e., mesor), impaired overall robustness (i.e., pseudo- $F$ statistics), and altered activity timing (i.e., acrophase), are associated with 1) poorer glycemic control (i.e., higher fasting glucose, insulin, and HOMA of insulin resistance [HOMA-IR]) and prevalent type 2 diabetes at baseline and 2) greater worsening in glycemic control and a higher risk for incident type 2 diabetes over 3.5 years of follow-up.

\section{RESEARCH DESIGN AND METHODS}

\section{Study Population}

The Osteoporotic Fractures in Men Study (MrOS) (https://mrosdata.sfcc-cpmc.net) is a multicenter cohort study of risk factors for osteoporosis and other aging outcomes in older men (19). Between 2000 and 2002, MrOS enrolled 5,994 communitydwelling, ambulatory men age $\geq 65$ years from six clinical centers in the U.S. (20). The Outcomes of Sleep Disorders in Older Men Study (MrOS Sleep study) is an MrOS ancillary study that included 3,135 MrOS participants with the aim to determine the relationships of sleep and restactivity rhythms with a broad set of health outcomes, including cardiovascular disease (CVD), cognitive decline, and falls and fractures (21). Between 2003 and 2005, the MrOS Sleep study collected objective measures of rest-activity data as well as fasting blood samples, and fasting blood samples were collected again between 2007 and 2009 during a follow-up visit for a subset of those from the baseline visit. Both the original MrOS study and ancillary studies were approved by the institutional review boards at each of the participating field sites (University of Alabama at Birmingham; University of Minnesota; Stanford University; University of California, San Diego; Oregon Health and Science University; University of Pittsburgh; and Case Western Reserve University), and written informed consent was obtained from each participant prior to enrollment.

Assessment of Rest-Activity Rhythms

Rest-activity rhythms were recorded using the Sleep-watch-O (Ambulatory Monitoring, Inc.) actigraph. Participants were instructed to wear the device continuously on the nondominant wrist for five 24-h periods. This actigraph contains a piezoelectric linear accelerometer, which generated a voltage each time the acceleration surpassed $0.003 \mathrm{~g}$. These voltages are gathered continuously and stored in 1-min epochs. Activity data were collected using the proportional integration mode. The orientation and sensitivity of the accelerometer are optimized for highly effective sleep-wake inference from wrist activity $(22,23)$. Rest-activity rhythm characteristics were derived by applying an extended cosine model analysis to the activity data. This method applies an antilogistic transformation to the cosine curve and fits activity data to a squared wave rather than a cosine curve (24). It has been reported that the extended cosine model is particularly useful for studying daily activity rhythms in the older population, whose activity patterns tend to deviate from a cosine shape (24). In this current analysis, we focused on five parameters: 1) amplitude measured as peak-to-nadir difference in activity of the fitted curve, an indicator of strength of rhythmicity; 2) mesor measured as minimum $+1 / 2$ amplitude, an indicator of average activity levels; 3 ) amplitudeto-mesor ratio or relative amplitude, a normalized measure of rhythm strength accounting for average activity levels; 4) pseudo-F statistics, a model goodnessof-fit measure and an indicator of overall rhythmicity; and 5) acrophase measured as the time of peak activity of the fitted curve. For amplitude, mesor, amplitudeto-mesor ratio, and pseudo-F statistics, we hypothesized that a higher value will be associated with better metabolic health and categorized each variable into quartiles. For acrophase, previous studies suggested a potential U-shaped association with some health outcomes including CVD (17) and we categorized it 
into three groups: early ( $<$ mean $-1 \mathrm{SD}$, before $1304 \mathrm{~h}$ ), normal (mean $\pm 1 \mathrm{SD}$, between $1304 \mathrm{~h}$ and $1529 \mathrm{~h}$ ), and late (>mean + 1 SD, after 1529 h). Quartiles and groups of rest-activity variables were derived using the entire sample of 3,058 men with valid rest and activity data.

Metabolic Biomarkers and Outcomes At both baseline and follow-up, fasting serum samples were collected in the morning, processed on the same day, and stored at $-70^{\circ} \mathrm{C}$. Baseline samples were collected on the same day when actigraphy recording started. Glucose was measured enzymatically with an interassay coefficient of variation of $<3 \%$, and insulin was measured by immunoenzymometric assay, with inter- and intra-assay coefficients of variation $<10 \%(25)$. HOMA-IR was calculated using the algorithm of Matthews et al. (26): fasting glucose (mg/ $\mathrm{dL}) \times$ fasting insulin $(\mu \mathrm{U} / \mathrm{mL}) / 405$. The distributions of baseline fasting glucose, insulin, and HOMA-IR were positively skewed, and we performed log transformation to approximate a normal distribution. We also calculated the changes in fasting glucose, insulin, and HOMA-IR between baseline and follow-up among those with repeated measures of glucose and insulin, with positive values indicating an increase in marker levels and negative values indicating a decrease. The distributions of the change variables for all three metabolic markers were approximately normal, and no transformation was performed. In addition to the continuous change variable, we also derived a binary variable to examine more substantial increases in metabolic markers as the outcome. For this binary variable, we assigned a value of 1 to those in the top $25 \%$ of the change variable for each marker (based on the prospective analytic sample [details below]), and the rest were assigned 0 .

At both baseline and follow-up, participants also reported history of chronic diseases including type 2 diabetes. In addition, participants were asked to bring in all prescription medications taken in the past 30 days to clinic visits for study coordinators to record information on medication use. We defined diabetes as fasting glucose $\geq 6.93 \mathrm{mmol} / \mathrm{L}$, self-reported prevalent diabetes, or use of hypoglycemic medications or insulin. We further defined impaired fasting glucose as fasting glucose $\geq 5.50$ and $<6.93 \mathrm{mmol} / \mathrm{L}$.

\section{Covariates}

Information on sociodemographic factors (age, education, race/ethnicity, marital status) and diet was self-reported at the enrollment of the parent study MrOS. Diet was measured using the Block 98 semiquantitative food-frequency questionnaire, which has previously been validated (27). A healthy diet score was calculated using factor analysis (28). Height and weight were measured at the baseline visit of the MrOS Sleep study using standard protocols, and we calculated BMI as weight in kilograms divided by the square of height in meters (2). Lifestyle habits measured in the MrOS included smoking, alcohol use, and physical activity. Physical activity was assessed using the Physical Activity Scale for the Elderly (PASE), a brief questionnaire specifically designed to assess physical activity levels in people age $\geq 65$ years. With PASE, we collected information on multiple occupational, household, and leisure activities and took into consideration both the frequency and duration of physical activity. A PASE composite score (range 0400) was calculated, and higher scores indicate higher activity levels (29). A validation study showed that PASE scores correlated well with energy expenditure (30). Self-rated health and medical history of chronic diseases including cancer and CVD and depression (Geriatric Depression Scale score $\geq 6$ [31]) were also assessed at the baseline visit of the MrOS Sleep study.

\section{Analytic Samples}

Figure 1 presents a flowchart for deriving analytic samples for various analyses in this study. Of the 5,994 men in the original MrOS cohort, 3,135 men enrolled in the MrOS Sleep study and provided objective data on rest-activity rhythms with use of 24-h actigraphy between 2003 and 2005, which is considered the baseline for our analysis. A total of 3,058 men had actigraphy data that passed quality control measures as previously described (21). Of these, we excluded those with missing information on rest-activity rhythms ( $N=$ 9) or metabolic markers ( $N=493$ ) and/or with extreme values (within the lowest or highest $1 \%[N=106])$ for fasting glucose, insulin, or HOMA-IR. The $\pm 1 \%$ cutoff points were chosen for removal of the extreme observations that could be due to measurement errors. The final analytic sample for cross-sectional analysis was 2,450 men. In sensitivity analysis, we further removed participants who were using hypoglycemic medications or insulin at baseline $(N=190)$.

A second fasting blood sample was collected at a follow-up visit (2007-2009), allowing for longitudinal analysis. A total of 973 participants from the cross-sectional analysis sample had fasting glucose and insulin levels for both the baseline and the follow-up assessments. Of these, we excluded 41 men who showed extreme (within the lowest or highest 1\%) changes in glucose, insulin, or HOMA-IR between baseline and follow-up, resulting in a sample of 932 men for prospective analysis for changes in metabolic markers. In the sensitivity analysis, we further removed participants using hypoglycemic medications or insulin at either baseline or follow-up $(N=110)$. Finally, for prospective analysis focusing on incident type 2 diabetes, we excluded those with prevalent diabetes at baseline $(n=76)$ from the 932 men included in the main analysis, resulting in a sample size of 861 .

\section{Statistical Analysis}

For descriptive analysis, we compared participant characteristics according to quartiles of amplitude and categories of acrophase using $\chi^{2}$ test (categorical variables), ANOVA (normally distributed variables), or Kruskal-Wallis test (continuous variables with nonnormal distributions). In addition to potential confounders listed below, we also compared important lifestyle and health factors, including $\mathrm{BMI}$, physical activity, sleep, self-rated health, cancer, depression, and CVD.

We used multiple linear regression to estimate adjusted geometric means (logtransformed baseline markers) and $\beta$-coefficient (change in markers) with 95\% Cls. We used multiple logistic regression to estimate odds ratios (ORs) for binary outcomes (diabetes vs. no diabetes, highest quartile of change vs. lower quartiles) and multinomial logistic regression to estimate relative risk ratio for categorical outcomes (diabetes, impaired fasting glucose, and no diabetes). For all regression analyses, we considered a series of models: model 1 included age as the only covariate. Model 2, which we consider our main model, included age (continuous) and other potential confounders, including study site (Birmingham, AL; Minneapolis, MN; Palo Alto, CA; Pittsburgh, PA; Portland, OR; San Diego, CA), education (less than high school, high 


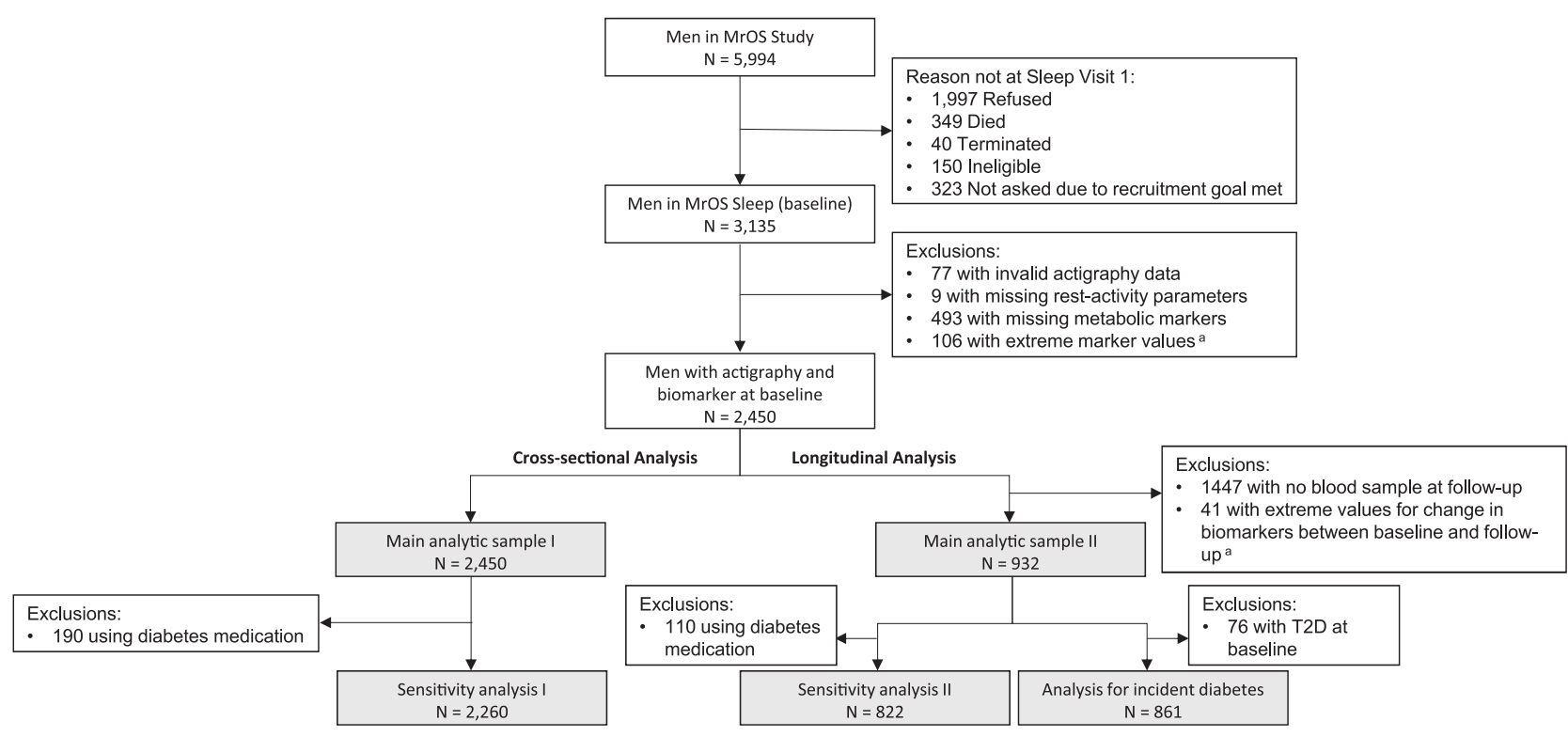

Figure 1-Flowchart showing the progression of participants. Gray-shaded rectangles show numbers for analytic samples for various analyses. ${ }^{a}$ Extreme values were defined as $<1 \%$ or $>99 \%$ of the sample distribution. T2D, type 2 diabetes.

school, some college, college, more than college), marital status (married, not married), race (White, non-White), smoking status (current, past, never), alcohol consumption ( $<1,1-13, \geq 14$ drinks/week), diet healthy index (quartiles), and season of data collection (December-February, March-May, June-August, SeptemberNovember). In a separate model, we additionally included measures of individual components (physical activity score and total sleep time, sleep efficiency, and midpoint of sleep [all as continuous variables]) of the rest-activity rhythm to assess whether the associations for rest-activity rhythms are independent of measures of individual behaviors. For the analysis focusing on incident diabetes, we included baseline HOMA-IR in a separate model (model 3) to assess to what degree the results may be driven by baseline predisposition to diabetes due to insulin resistance. We also considered variables that are potential mediators (BMI) or colliders (health outcomes including history of CVD, cancer, and depression at baseline), but because adjusting for mediators and colliders is analytically problematic $(32,33)$, we omitted these covariates in the main analysis. To test for trend, we modeled categorical variables as ordinal and evaluated this coefficient using the Wald test. Although we performed regressions for multiple exposure and outcome variables and false positives are a concern, many of our variables were highly correlated and some of the variables were a direct function of other variables (e.g., HOMA-IR is derived from fasting glucose and insulin), and we did not control for multiple comparisons using conventional methods such as Bonferroni corrections because such methods assumes independence among tests (34). More importantly, as Greenland et al. (35) pointed out, overemphasis of measures of statistical significance such as $P$ values can be misleading. Therefore, we avoided making judgement based on statistical significance and instead presented Cls and $P$ values for all the associations and evaluated our results based on both effect sizes and measures of statistical significance.

\section{RESULTS}

Study participant characteristics are presented in Table 1, according to amplitude and acrophase. We found that when compared with men in the highest quartile of amplitude, men with a lower amplitude were older, had higher BMI, and were more likely to report poorer self-rated health; lower physical activity, alcohol intake, and sleep efficiency; later sleep midpoint; and higher prevalence of CVD, depression, and cancer. When compared with normal acrophase, both early and late acrophases were associated with lower education level, nonmarried status, and less alcohol intake. Late acrophase was also associated with lower physical activity and sleep efficiency compared with normal and early acrophase groups. Finally, there was a strong positive correlation between acrophase and midpoint of sleep.

In cross-sectional analysis, amplitude, mesor, amplitude-to-mesor ratio, and pseudo-F statistic were all inversely associated with fasting insulin and HOMA-IR $\left(P_{\text {trend }}<0.0001\right.$ for all associations) (Table 2). Adjustment for multiple confounders had minimal impact on the findings (model 2 in Table 2). In contrast, the associations between rest-activity rhythm parameters and fasting glucose were generally weak and not statistically significant. In addition, we did not observe any association between acrophase and metabolic markers. After exclusion of participants using hypoglycemic medications or insulin, we observed little change to the results (no medication column in Table 2). These results were minimally impacted by additional adjustment for physical activity and sleep variables (Supplementary Table 1) or by exclusion of participants with chronic conditions (CVD, cancer, and depression) at baseline (data not shown).

Next, we examined rest-activity rhythms in relation to prevalent diabetes at baseline, and results are presented in Table 3. We found a trend supporting a relationship between lower quartile of amplitude and higher odds of type 2 diabetes $\left(\mathrm{OR}_{\mathrm{Q} 1}\right.$ vs. Q4 $1.63[95 \% \mathrm{Cl} 1.14,2.30], P_{\text {trend }}=0.01$ ). Moreover, a late acrophase was also associated with higher odds of diabetes in comparison with the normal group (OR $1.46[95 \% \mathrm{Cl} 1.04,2.04])$. Analysis using the 
Table 1-Baseline (2003-2005) characteristics of older men according to quartiles of amplitude and categories of acrophase in the MrOS Sleep study

\begin{tabular}{|c|c|c|c|c|c|c|c|c|c|}
\hline & \multicolumn{4}{|c|}{ Amplitude } & \multirow[b]{2}{*}{$P$} & \multicolumn{3}{|c|}{ Acrophase $^{a}$} & \multirow[b]{2}{*}{$P$} \\
\hline & Q1 & Q2 & Q3 & Q4 & & Early & Normal & Late & \\
\hline Age, years & $78.1(5.9)$ & $76.8(5.5)$ & $75.8(5.2)$ & $74.8(4.9)$ & $<0.0001^{\mathrm{b}}$ & $76.7(5.3)$ & $76.2(5.5)$ & $76.6(5.7)$ & $0.28^{\mathrm{b}}$ \\
\hline $\begin{array}{l}\text { Education: college } \\
\text { or higher }\end{array}$ & 53.2 & 60.1 & 56.1 & 53.0 & $0.07^{c}$ & 45.4 & 58.2 & 54.0 & $0.0002^{c}$ \\
\hline Married & 79.8 & 83.6 & 86.4 & 85.4 & $0.01^{\mathrm{c}}$ & 82.3 & 85.3 & 77.0 & $0.0007^{c}$ \\
\hline White & 91.5 & 93.1 & 92.9 & 89.1 & $0.04^{c}$ & 94.3 & 91.3 & 90.4 & $0.13^{c}$ \\
\hline $\begin{array}{l}\text { Smoking: former } \\
\text { or current }\end{array}$ & 61.6 & 56.8 & 59.3 & 62.3 & $0.47^{c}$ & 63.1 & 59.4 & 59.7 & $0.07^{c}$ \\
\hline $\begin{array}{l}\text { Alcohol: } \geq 1 \text { drink/ } \\
\text { week }\end{array}$ & 46.9 & 54.0 & 51.9 & 59.4 & $<0.0001^{\mathrm{c}}$ & 47.7 & 55.1 & 48.1 & $0.008^{c}$ \\
\hline Healthy diet score & $-0.06(1.02)$ & $0.02(1.05)$ & $0.06(1.04)$ & $0.05(0.98)$ & $0.10^{b}$ & $-0.01(1.02)$ & $0.02(1.02)$ & $0.03(1.01)$ & $0.89^{b}$ \\
\hline BMI & $27.9(4.0)$ & $27.2(3.6)$ & $26.8(3.5)$ & $26.7(3.56)$ & $<0.0001^{\mathrm{b}}$ & $27.6(3.9)$ & $27.0(3.6)$ & $27.3(3.9)$ & $0.04^{b}$ \\
\hline $\begin{array}{l}\text { Self-rated health: } \\
\text { good/excellent }\end{array}$ & 79.6 & 86.3 & 92.0 & 90.5 & $<0.0001^{\mathrm{c}}$ & 86.0 & 88.2 & 82.8 & $0.02^{c}$ \\
\hline $\begin{array}{l}\text { Physical activity } \\
\text { score }\end{array}$ & $117.9(64.4)$ & $139.2(65.0)$ & $157.3(68.0)$ & $172.0(72.8)$ & $<0.0001^{d}$ & $151.2(73.5)$ & $149.6(69.6)$ & $127.9(70.4)$ & $<0.0001^{d}$ \\
\hline Total sleep time, $\mathrm{h}$ & $6.3(1.5)$ & $6.5(1.1)$ & $6.5(1.1)$ & $6.4(1.1)$ & $0.08^{d}$ & $6.3(1.2)$ & $6.4(1.2)$ & $6.3(1.2)$ & $0.003^{d}$ \\
\hline Sleep efficiency & $0.75(0.15)$ & $0.79(0.11)$ & $0.80(0.10)$ & $0.79(0.11)$ & $<0.0001^{d}$ & $0.79(0.12)$ & $0.79(0.12)$ & $0.76(0.12)$ & $0.005^{d}$ \\
\hline Sleep midpoint & $\begin{array}{l}0301 \mathrm{~h} \\
\text { (59 min) }\end{array}$ & $\begin{array}{l}0248 \mathrm{~h} \\
(57 \mathrm{~min})\end{array}$ & $\begin{array}{l}0247 \mathrm{~h} \\
(52 \mathrm{~min})\end{array}$ & $\begin{array}{l}0248 \mathrm{~h} \\
\text { (55 min) }\end{array}$ & $<0.0001^{d}$ & $\begin{array}{c}0155 \mathrm{~h} \\
\text { (59 min) }\end{array}$ & $\begin{array}{l}0248 \mathrm{~h} \\
(40 \mathrm{~min})\end{array}$ & $\begin{array}{c}0410 \mathrm{~h} \\
(42 \mathrm{~min})\end{array}$ & $<0.0001^{\mathrm{d}}$ \\
\hline Disease history & & & & & & & & & \\
\hline CVD & 43.2 & 37.7 & 34.5 & 30.2 & $<0.0001^{\mathrm{c}}$ & 36.9 & 35.3 & 41.5 & $0.10^{c}$ \\
\hline Depression & 10.2 & 5.8 & 4.6 & 4.8 & $0.0001^{c}$ & 6.0 & 5.8 & 9.3 & $0.07^{c}$ \\
\hline Cancer & 29.3 & 30.8 & 30.2 & 22.6 & $0.004^{c}$ & 28.6 & 28.3 & 26.8 & $0.85^{\mathrm{c}}$ \\
\hline
\end{tabular}

Data are \% or mean (SD). ${ }^{a}$ Acrophase was categorized as early (mean - 1 SD, before $1304 \mathrm{~h}$ ), normal (mean \pm 1 SD, between $1304 \mathrm{~h}$ and $1529 \mathrm{~h}$ ), and late (mean +1 SD, after $1529 \mathrm{~h}$ ). ${ }^{\mathrm{b}} P$ values were derived from ANOVA. ${ }^{c} P$ values were derived from $\chi^{2}$ test. ${ }^{d} P$ values were derived from Kruskal-Wallis test.

three-category outcome variable (diabetes, impaired fasting glucose, and normoglycemia group) showed similar findings for diabetes, but results for impaired fasting glucose were largely null (Supplementary Table 2).

In prospective analysis focusing on changes in metabolic markers as continuous outcomes, we found no association between any of the rest-activity rhythm characteristics and changes in fasting glucose, insulin, or HOMA-IR (Table 4). Exclusion of participants who reported taking hypoglycemic medications or insulin at either baseline or follow-up had little impact on the results. However, when we examined change in markers as a binary outcome to focus on more substantial increases in marker levels (an increase within the top $25 \%$ ), we found evidence suggesting an association of lower amplitude and mesor with a substantial increase in HOMA-IR over follow-up ( $P_{\text {trend }}=0.009$ for amplitude and 0.03 for mesor) (Supplementary Table 3). We also found that early acrophase was associated with $91 \%$ higher odds of a substantial increase in HOMA-IR over follow-up (OR 1.91 [95\% Cl 1.24, 2.95]).
Finally, we examined rest-activity rhythm characteristics and incident diabetes among men who were free of type 2 diabetes at baseline (Table 5). Despite a small number of people who developed incident diabetes ( $n=48)$, the results seem to support a relationship between lower amplitude and a higher risk of developing diabetes. When compared with men in the highest quartile of amplitude, men in the lowest quartile were more than three times more likely to develop diabetes at follow-up (OR 3.81 [95\% Cl 1.45, 10.00], $P=0.001$ ). Similar trends were also observed for amplitudeto-mesor ratio (OR $2.79[95 \% \mathrm{Cl} 1.10$, 7.07], $P=0.03$ ), and pseudo-F (OR 3.49 $[95 \% \mathrm{Cl} 1.34,9.10], P=0.006)$, indicating that this association was a reflection not just of overall activity but also of the strength of day-night rhythmicity. We also observed an association between late acrophase (compared with normal) and higher risk of incident diabetes (OR 2.44 [95\% Cl 1.09, 5.47]). Additional adjustment for baseline HOMA-IR (model 3 in Table 5) attenuated the results, but the trends remained similar, suggesting that the observed association could not be fully explained by baseline differences in insulin resistance.

\section{CONCLUSIONS}

In a cohort of older men, we found that multiple characteristics of rest-activity rhythms, particularly lower amplitude, mesor, amplitude-to-mesor ratio, and overall rhythmicity, were associated with elevated fasting insulin and higher insulin resistance at baseline. Moreover, a lower amplitude and amplitude-to-mesor ratio, reduced overall rhythmicity, and a late acrophase were also found to predict higher risk of developing type 2 diabetes over $\sim 3.5$ years follow-up. However, the relatively small number of incident diabetes cases in this population warrants confirmation in future studies.

The cross-sectional results from this study are consistent with previous investigations on rest-activity rhythms and metabolic health using 24-h actigraphy data, which also suggested that impaired rest-activity rhythms are associated with metabolic disorders. For example, in two studies of older participants, the Rush Memory and Aging Project in the U.S. and the Rotterdam Study, researchers found 


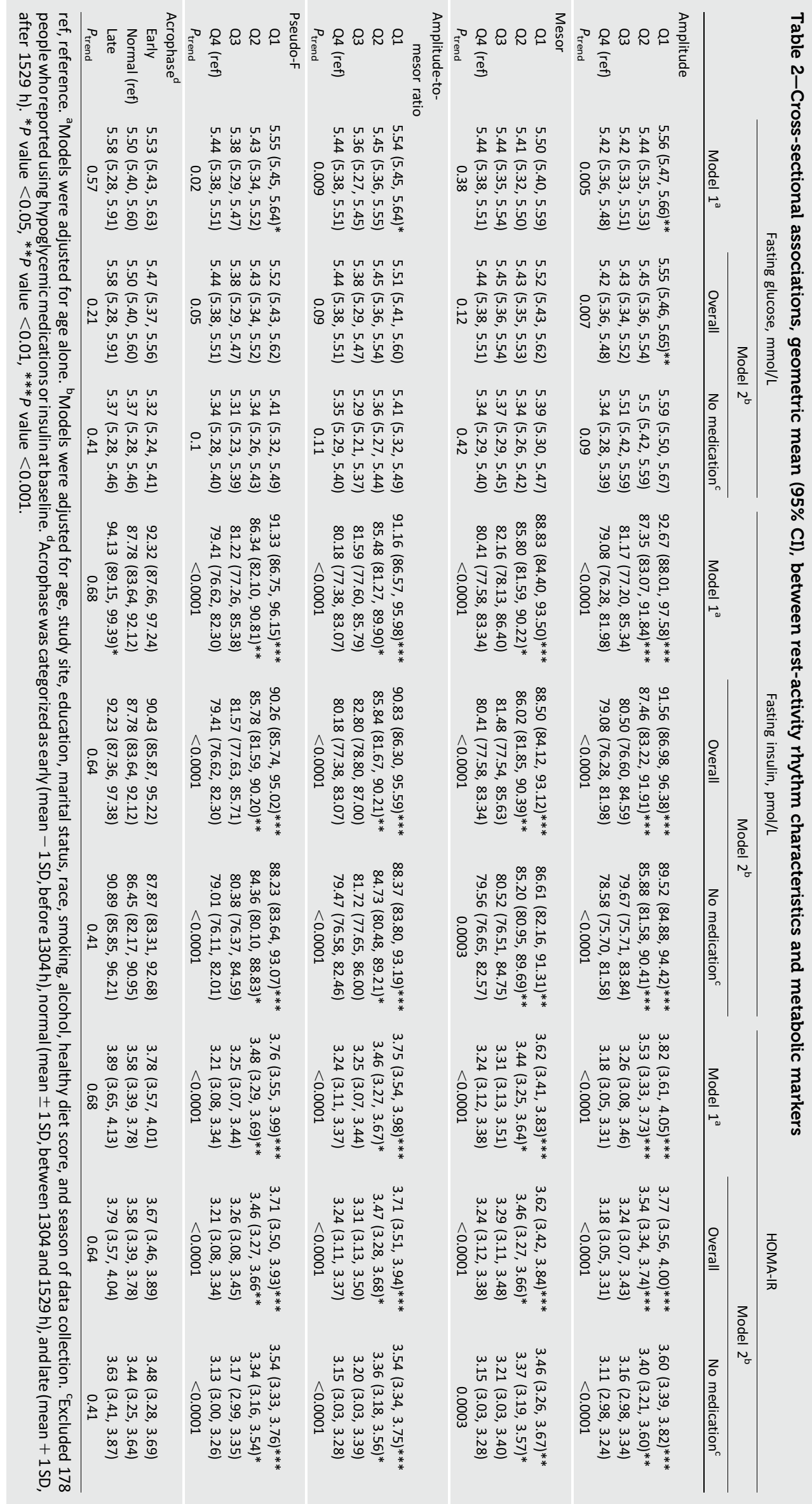


Table 3-Cross-sectional associations between rest-activity rhythm characteristics and type 2 diabetes

\begin{tabular}{|c|c|c|c|}
\hline & \multirow{2}{*}{$\begin{array}{c}n(\%) \text { with } \\
\text { diabetes }^{a}\end{array}$} & \multicolumn{2}{|c|}{ OR $(95 \% \mathrm{Cl})$} \\
\hline & & Model $1^{\mathrm{b}}$ & Model $2^{c}$ \\
\hline \multicolumn{4}{|l|}{ Amplitude } \\
\hline Q1 & $97(16.5)$ & $1.68(1.20,2.36)^{* *}$ & $1.63(1.14,2.30)^{* *}$ \\
\hline Q2 & $79(12.7)$ & $1.21(0.86,1.71)$ & $1.23(0.87,1.75)$ \\
\hline Q3 & $78(12.8)$ & $1.20(0.85,1.70)$ & $1.21(0.85,1.72)$ \\
\hline Q4 & 70 (11.1) & ref & Ref \\
\hline$P_{\text {trend }}$ & & 0.004 & 0.01 \\
\hline \multicolumn{4}{|l|}{ Mesor } \\
\hline Q1 & $90(15.2)$ & $1.33(0.96,1.85)$ & $1.41(1.00,1.98)$ \\
\hline Q2 & 77 (12.5) & $1.04(0.74,1.46)$ & $1.10(0.78,1.55)$ \\
\hline Q3 & $80(13.2)$ & $1.10(0.79,1.54)$ & $1.11(0.79,1.57)$ \\
\hline Q4 & $77(12.2)$ & ref & Ref \\
\hline$P_{\text {trend }}$ & & 0.13 & 0.07 \\
\hline \multicolumn{4}{|c|}{$\begin{array}{l}\text { Amplitude-to-mesor } \\
\text { ratio }\end{array}$} \\
\hline Q1 & $96(16.4)$ & $1.49(1.07,2.07)^{* *}$ & $1.34(0.96,1.88)$ \\
\hline Q2 & $81(13.0)$ & $1.12(0.89,1.57)$ & $1.10(0.78,1.55)$ \\
\hline Q3 & 72 (11.6) & $0.96(0.68,1.36)$ & $1.01(0.71,1.43)$ \\
\hline Q4 & 75 (12.1) & ref & Ref \\
\hline$P_{\text {trend }}$ & & 0.01 & 0.08 \\
\hline \multicolumn{4}{|l|}{ Pseudo-F } \\
\hline Q1 & 98 (16.7) & $1.44(1.04,1.99)^{* *}$ & $1.34(0.96,1.84)$ \\
\hline Q2 & $78(12.7)$ & $1.02(0.73,1.43)$ & $1.00(0.71,1.41)$ \\
\hline Q3 & $69(11.1)$ & $0.87(0.61,1.22)$ & $0.84(0.59,1.19)$ \\
\hline Q4 & $79(12.7)$ & ref & Ref \\
\hline$P_{\text {trend }}$ & & 0.02 & 0.05 \\
\hline \multicolumn{4}{|c|}{ Acrophase $^{d}$} \\
\hline Early & 52 (14.9) & $1.27(0.92,1.76)$ & $1.11(0.79,1.56)$ \\
\hline Normal & $217(12.1)$ & ref & ref \\
\hline Late & 55 (17.6) & $1.55(1.12,2.14)^{*}$ & $1.46(1.04,2.04)^{*}$ \\
\hline$P_{\text {trend }}$ & & 0.37 & 0.23 \\
\hline
\end{tabular}

${ }^{\text {a }}$ Represents \% of participants in the quartile or category of the rest-activity rhythm characteristic who had diabetes at baseline. ${ }^{b}$ Models were adjusted for age alone. ${ }^{c}$ Models were adjusted for age, study site, education, marital status, race, smoking, alcohol, healthy diet score, and season of data collection. ${ }^{\mathrm{d}}$ Acrophase was categorized as early (mean - 1 SD, before $1304 \mathrm{~h}$ ), normal (mean \pm $1 \mathrm{SD}$, between 1304 and $1529 \mathrm{~h}$ ), and late (mean +1 SD, after $1529 \mathrm{~h}$ ). $* p$ value $<0.05, * * p$ value $<0.01$

that impaired day-to-day regularity (measured by interdaily stability) and increased fragmentation (measured by intraday variability) of rest-activity rhythms were associated with a wide range of metabolic outcomes, including obesity, metabolic syndrome, hypertension, type 2 diabetes, and dyslipidemia $(15,16)$. In addition, a smaller study of 578 middle-aged adults (average age 52 years) found that a lower relative amplitude (the difference in activity between the highest and lowest periods divided by their sum, similar to amplitude-to-mesor ratio) was associated with higher BMI (36). In our study, we extended previous studies by reporting the associations between additional restactivity characteristics and metabolic outcomes related to glucose control. Specifically, we found that multiple characteristics of rest-activity rhythms were associated with fasting insulin and HOMA-IR. The associations appeared to be weaker for fasting glucose, which may be explained by $\beta$-cell compensation that leads to increased insulin secretion to keep a stable blood glucose level in the presence of insulin resistance (37). This often occurs in the early stage of impaired glucose control (i.e., in people with prediabetes). Therefore, the consistent and highly significant results for insulin and HOMA-IR suggest that disrupted rest-activity rhythms were associated with insulin resistance in older men. Despite a variety of approaches used to analyze 24-h rest-activity rhythms, from nonparametric measures such as interdaily stability and intraday variability reported previously to parametric measures in our study derived from extended cosine models, almost all of the rest-activity rhythm characteristics associated with adverse metabolic outcomes indicate weakened rhythmicity (e.g., lower interdaily stability, higher intraday variability, lower amplitude and amplitude-to-mesor ratio, and reduced pseudo-F statistics). Taken together, findings from previous studies and this current study support our hypothesis that less robust rest-activity rhythms are associated with adverse metabolic outcomes. However, the temporal ambiguity of cross-sectional associations makes it challenging to determine whether impaired rhythmicity is a risk factor or a symptom of metabolic dysfunction.

Results from our prospective analyses are mixed. On the one hand, we found little evidence supporting an association between baseline rest-activity rhythms and prospective changes in fasting glucose, insulin, and HOMA-IR. On the other hand, we found that a lower amplitude, a lower amplitude-to-mesor ratio (relative amplitude, adjusted for mean activity), reduced overall rhythm robustness, and a later acrophase were significant predictors for the risk of developing incident type 2 diabetes over follow-up. One potential explanation for the discrepancies between the results from the two different analyses is that the relationships between rest-activity characteristics and changes in biomarker levels are not linear; instead, rest-activity characteristics may have a stronger association with more extreme changes in metabolic markers that may result in the development of diabetes. To test this hypothesis, we examined the associations between rest-activity rhythm characteristics and the likelihood of having a substantial increase (i.e., an increase in the upper $25 \%$ in our study population) in metabolic markers. Indeed, we found evidence supporting a relationship between lower amplitude and mesor and substantial increases in HOMA-IR over follow-up, which is consistent with a potential role of weakened rest-activity rhythms as a risk factor for type 2 diabetes.

We also found that an early acrophase was associated with higher odds of substantial increase in fasting glucose, insulin, and HOMA-IR. Acrophase is a measure of the timing of peak activity and is also correlated with sleep timing in our study (spearman correlation coefficient, 0.71 ). There is a growing literature suggesting that a later sleep timing is associated with impaired glycemic control $(38,39)$. However, the midpoint of sleep was not significantly associated 


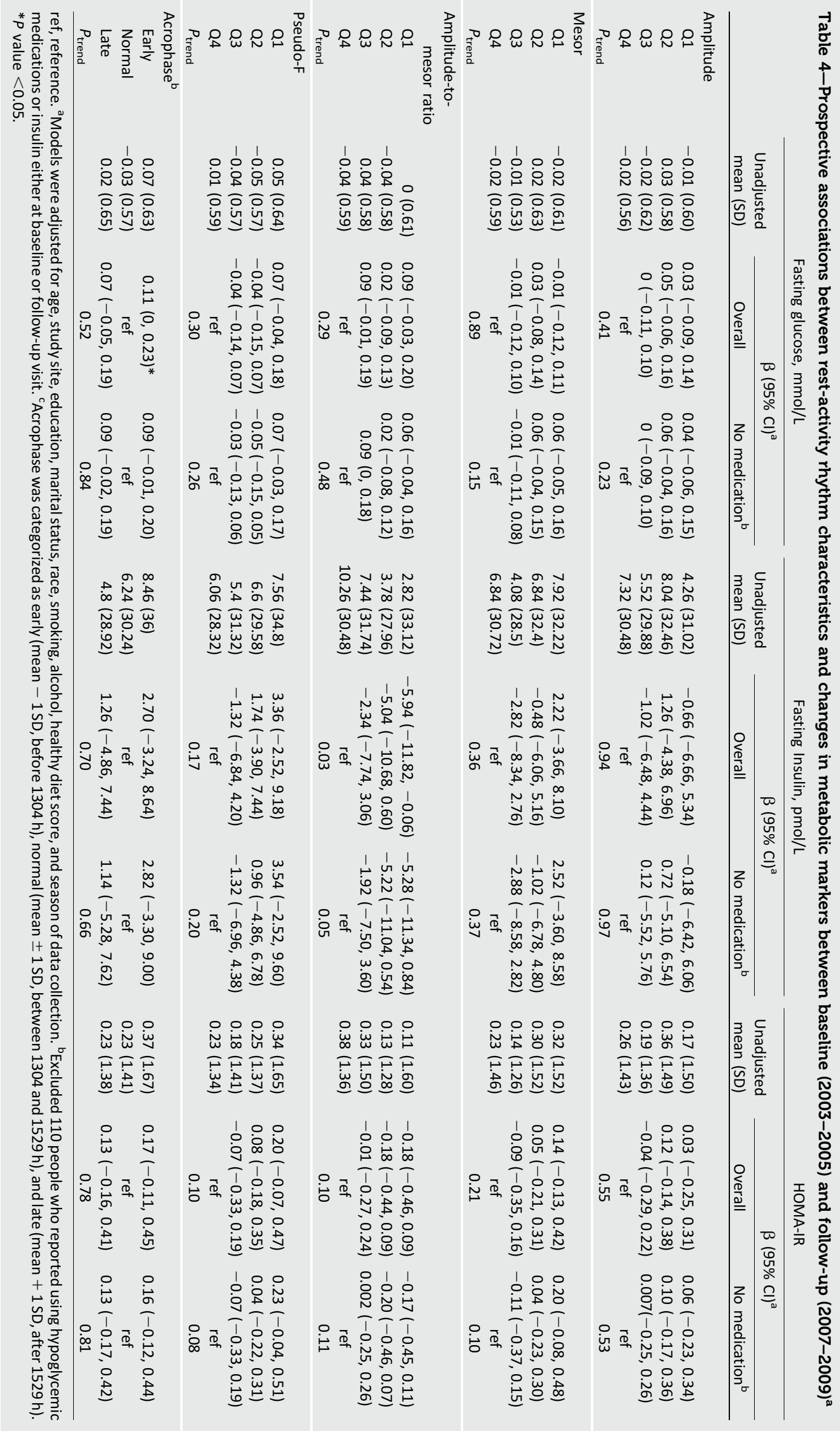


Table 5-Prospective associations between rest-activity rhythm characteristics and incident type 2 diabetes among subjects without diabetes at baseline ${ }^{\mathrm{a}}$

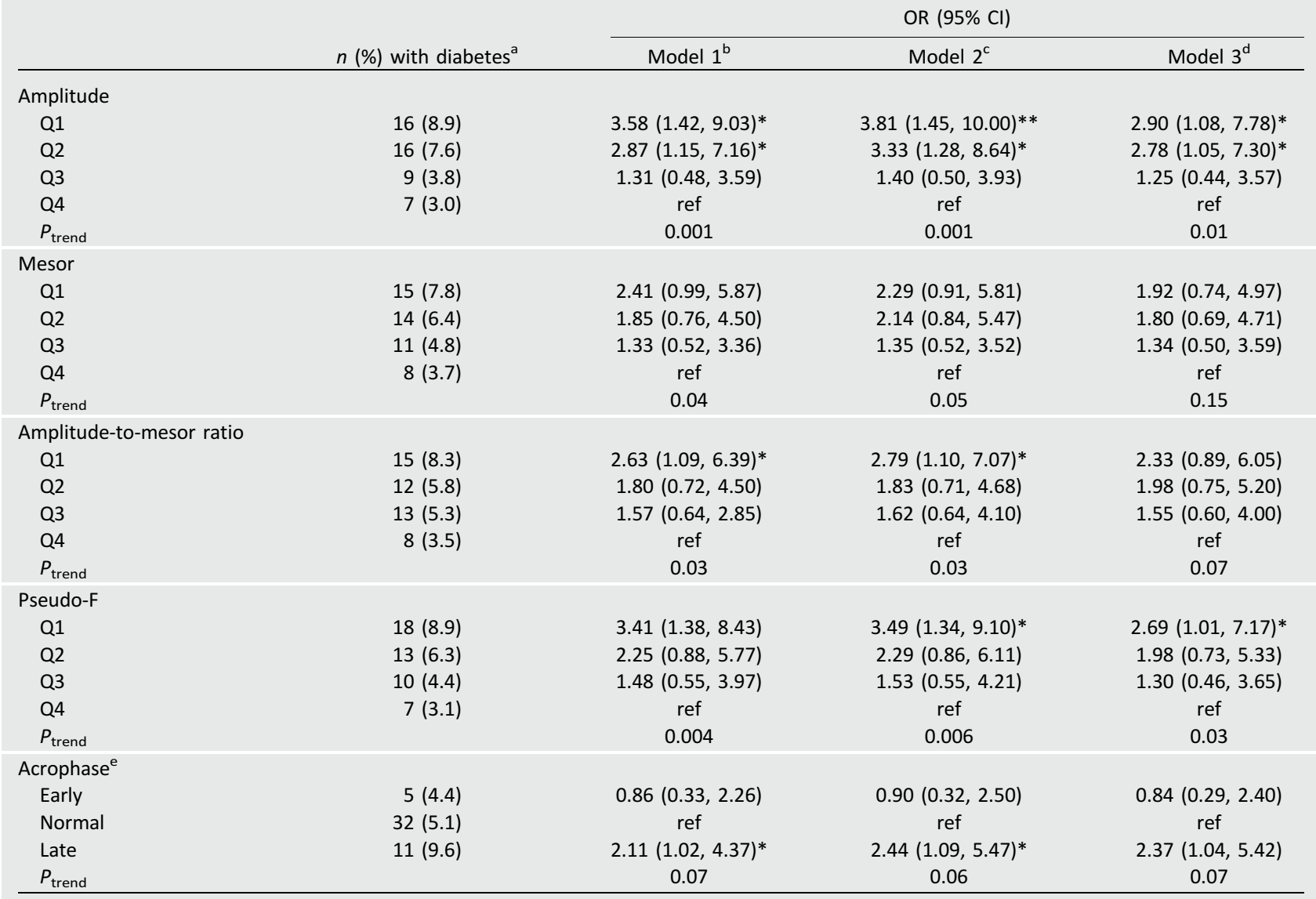

${ }^{\mathrm{a}}$ Represents \% of participants in the quartile or category of the rest-activity rhythm characteristic who developed diabetes over follow-up. ${ }^{\mathrm{b}}$ Models were adjusted for age alone. 'Models were adjusted for age, study site, education, marital status, race, smoking, alcohol, healthy diet score, and season of data collection. ${ }^{\mathrm{d}}$ Models were adjusted for variables in model 2 and baseline HOMA-IR. ${ }^{\mathrm{e}}$ Acrophase was categorized as early (mean - $1 \mathrm{SD}$, before $1304 \mathrm{~h}$ ), normal (mean $\pm 1 \mathrm{SD}$, between 1304 and $1529 \mathrm{~h}$ ), and late (mean $+1 \mathrm{SD}$, after $1529 \mathrm{~h}$ ). ${ }^{*} p$ value $<0.05,{ }^{* *} p$ value $<0.01$.

with metabolic markers in our study, and additionally controlling for sleep timing had little impact on the findings, suggesting that the relationships between acrophase and HOMA-IR and incident diabetes cannot be explained by the effects of sleep timing. Alternatively, timing of daytime physical activity has also been suggested to play a role in metabolic health (40), and several recent studies reported that evening exercise may be more effective in lowering glucose levels than morning exercise $(41,42)$. However, it is worth nothing that we observed contradictory results for the association between acrophase and incident diabetes, where a later acrophase was associated with a higher risk of type 2 diabetes. Due to limited sample sizes, particularly small numbers of incident diabetes cases (only 5 and 11 participants in the early and late acrophase groups), the relationship between acrophase and metabolic health needs to be examined in future prospective studies.
The observed relationship between altered rest-activity rhythms and metabolic dysfunction may be explained by three mechanisms: First, weakened rest-activity rhythms cause metabolic dysfunction. This possibility is supported by numerous animal and human experimental studies demonstrating that disruption of circadian rhythms, a main influence on the overall pattern of 24-h rest-activity rhythms, may cause metabolic dysfunction (43). For example, disruption of the circadian clock through mutations in core circadian genes leads to impaired $\beta$-cell function and diabetes in mice (44). In human subjects, laboratory-induced circadian misalignment caused impaired glucose tolerance and reduced insulin sensitivity $(45,46)$. However, it is worth noting that in our study, we did not have measurements of internal circadian rhythms, and it is unclear whether the observed associations reflected the adverse effects of an impaired internal circadian rhythm or were primarily driven by altered behaviors themselves. Second, metabolic dysfunction may also impact rest-activity rhythms. For example, obesity is a well-established risk factor for sleep apnea (47), which disrupts sleep and increases sleep fragmentation and may lead to daytime sleepiness and fatigue that may also prevent individuals from engaging in exercise and other physical activities, all of which could lead to altered rest-activity patterns. In our prospective analysis focusing on incident diabetes, we observed that among men without diabetes at baseline, rest-activity rhythm characteristics predicted future development of diabetes, suggesting that impairment in rest-activity rhythms contributed to the development of metabolic dysfunction. However, it is likely that other forms of subclinical metabolic dysfunction were present at baseline, and we cannot exclude the possibility that our results were driven by the impact of metabolic dysfunction on rest-activity patterns. Finally, it is also 
possible that common processes, likely associated with aging, cause both alterations in rest-activity rhythms and metabolic dysfunction. It is worth noting that the three explanations are not necessarily mutually exclusive, and future studies should focus on elucidating the multiple mechanisms that may drive the relationship between rest-activity rhythms and metabolic health. In particular, we encourage intervention studies to examine the potential benefits of strategies to enhance rest-activity rhythms, such as timed light exposure, carefully designed exercise and meal schedules, and improved sleep hygiene. Findings from such studies not only will help establish (or refute) a causal relationship between rest-activity rhythms and metabolic health but also will offer valuable evidence for clinical practice. On the other hand, our prospective results suggest that, regardless of the nature and direction of the relationship between restactivity rhythms and metabolic health, multiple characteristics of 24-h rest-activity may serve as unique predictors of metabolic dysfunction, which should be considered by future studies that aim at developing prediction models for risks of cardiometabolic diseases.

Our study has a number of important strengths. First of all, the repeated assessments of biomarkers at both baseline and follow-up have enabled us to examine both the cross-sectional and prospective relationships between rest-activity rhythms and metabolic health. Second, we used 24-h actigraphy data to objectively measure multiple characteristics of rest-activity rhythms using an extended cosine model, an approach that is appropriate for assessing activity patterns of the older population (24). Third, we were able to assess several related yet distinct indicators of metabolic health, and our assessment of diabetes incorporated information from both selfreport data and objectively measured biomarkers. Our study also has several limitations. First, the number of cases of incident diabetes was limited, probably due to a relatively short follow-up (3.5 years). Future studies with a larger sample size and longer follow-up are needed to clarify the association between restactivity characteristics and risk of diabetes. In addition, rest-activity rhythms are shaped by both the internal circadian clock and environmental factors such as constraints on daily schedules. We did not have measures of internal circadian rhythms, such as dim light melatonin onset, and we also did not have detailed information on participants' daily schedules or information on light exposure patterns and therefore were unable to further examine the contribution of both internal and external factors to the relationships between rest-activity rhythms and metabolic health. Finally, our group of study participants was all older men and was predominantly White, which limited the ability to generalize the findings to women, younger populations, and racial/ethnic minority groups. For example, it has been reported that men and women may have different rest-activity patterns $(13,14)$ and different metabolic responses to circadian alterations (48); therefore, future studies are needed to examine the association of rest-activity rhythms with metabolic health in women.

In conclusion, our study found that weakened rest-activity rhythms are associated with indicators of impaired metabolic function and predict higher risk for incident diabetes. The findings, although they need to be confirmed by future prospective investigations, contribute to a growing body of literature suggesting an important role of rest-activity rhythms in metabolic health. Future studies should also focus on pinpointing the underlying mechanisms linking rest-activity rhythms with metabolic health, testing interventions targeting regulating rest-activity rhythms to improve metabolic health, and incorporating characteristics of restactivity rhythms into algorithms to better predict metabolic disease risks.

Funding. MrOS is supported by National Institutes of Health (NIH) funding. The following institutes provide support: the National Institute on Aging, the National Institute of Arthritis and Musculoskeletal and Skin Diseases, the National Center for Advancing Translational Sciences, and $\mathrm{NIH}$ Roadmap for Medical Research under the following grant numbers: U01 AG027810, U01 AG042124, U01 AG042139, U01 AG042140, U01 AG042143, U01 AG042145, U01 AG042168, U01 AR066160, and UL1 TR000128. The National Heart, Lung, and Blood Institute provides funding for the MrOS Sleep ancillary study under the following grant numbers: R01 HL071194, R01 HL070848, R01 HL070847, R01 HL070842, R01 HL070841, R01 HL070837, R01 HL070838, and R01 HL070839.

Duality of Interest. No potential conflicts of interest relevant to this article were reported. Author Contributions. Q.X. designed the study, conducted data analysis, and drafted the manuscript. J.Q., F.A.J.L.S., and K.S. provided input on data analysis and results interpretation and commented on the manuscript. D.S.E., S.R., N.E.L., and S.A.-I. reviewed and edited the manuscript. Q.X. and K.S. developed the study concept and design. Q.X. performed statistical analysis. Q.X, J.Q., F.A.J.L.S., and K.S. interpreted data. Q.X., J.Q., D.S.E., S.R., N.E.L., S.A.-I., F.A.J.L.S., and K.S. critically revised the manuscript for important intellectual content. Q.X. is the guarantor of this work and, as such, had full access to all the data in the study and takes responsibility for the integrity of the data and the accuracy of the data analysis.

\section{References}

1. Lambert EV, Bull F. Public health recommendations for physical activity in the prevention of type 2 diabetes mellitus. Med Sport Sci 2014;60: 130-140

2. $\mathrm{Wu} \mathrm{Y,} \mathrm{Zhai} \mathrm{L,} \mathrm{Zhang} \mathrm{D.} \mathrm{Sleep} \mathrm{duration} \mathrm{and}$ obesity among adults: a meta-analysis of prospective studies. Sleep Med 2014;15:1456-1462 3. Kinuhata S, Hayashi T, Sato KK, et al. Sleep duration and the risk of future lipid profile abnormalities in middle-aged men: the Kansai Healthcare Study. Sleep Med 2014;15:1379-1385

4. Huang T, Redline S. Cross-sectional and prospective associations of actigraphy-assessed sleep regularity with metabolic abnormalities: the Multi-Ethnic Study of Atherosclerosis. Diabetes Care 2019;42:1422-1429

5. Cappuccio FP, D’Elia L, Strazzullo P, Miller MA Quantity and quality of sleep and incidence of type 2 diabetes: a systematic review and metaanalysis. Diabetes Care 2010;33:414-420

6. Gan Y, Yang C, Tong $X$, et al. Shift work and diabetes mellitus: a meta-analysis of observational studies. Occup Environ Med 2015;72:72-78

7. Hood S, Amir S. The aging clock: circadian rhythms and later life. J Clin Invest 2017;127: 437-446

8. Duffy JF, Zitting KM, Chinoy ED. Aging and circadian rhythms. Sleep Med Clin 2015;10: 423-434

9. Duffy JF, Dijk DJ, Klerman EB, Czeisler CA. Later endogenous circadian temperature nadir relative to an earlier wake time in older people. Am J Physiol 1998;275:R1478-R1487

10. Duffy JF, Zeitzer JM, Rimmer DW, Klerman EB, Dijk DJ, Czeisler CA. Peak of circadian melatonin rhythm occurs later within the sleep of older subjects. Am J Physiol Endocrinol Metab 2002;282:E297-E303

11. Roenneberg $T$, Kuehnle $T$, Juda $M$, et al. Epidemiology of the human circadian clock. Sleep Med Rev 2007;11:429-438

12. Ohayon MM, Carskadon MA, Guilleminault C, Vitiello MV. Meta-analysis of quantitative sleep parameters from childhood to old age in healthy individuals: developing normative sleep values across the human lifespan. Sleep 2004;27: 1255-1273

13. Unruh ML, Redline S, An MW, et al. Subjective and objective sleep quality and aging in the sleep heart health study. J Am Geriatr Soc 2008;56:1218-1227

14. van den Berg JF, Miedema HM, Tulen JH, Hofman A, Neven AK, Tiemeier H. Sex differences in subjective and actigraphic sleep measures: a population-based study of elderly persons. Sleep 2009;32:1367-1375 
15. Sohail S, Yu L, Bennett DA, Buchman AS, Lim AS. Irregular 24-hour activity rhythms and the metabolic syndrome in older adults. Chronobiol Int 2015;32:802-813

16. Luik Al, Zuurbier LA, Hofman A, Van Someren EJ, Tiemeier $\mathrm{H}$. Stability and fragmentation of the activity rhythm across the sleep-wake cycle: the importance of age, lifestyle, and mental health. Chronobiol Int 2013;30:1223-1230

17. Paudel ML, Taylor BC, Ancoli-Israel S, et al. Rest/activity rhythms and cardiovascular disease in older men. Chronobiol Int 2011;28:258-266 18. Tranah GJ, Blackwell T, Ancoli-Israel S, et al.; Study of Osteoporotic Fractures Research Group. Circadian activity rhythms and mortality: the study of osteoporotic fractures. J Am Geriatr Soc 2010;58:282-291

19. Blank JB, Cawthon PM, Carrion-Petersen ML, et al. Overview of recruitment for the osteoporotic fractures in men study (MrOS). Contemp Clin Trials 2005;26:557-568

20. Orwoll E, Blank JB, Barrett-Connor E, et al. Design and baseline characteristics of the osteoporotic fractures in men (MrOS) study-a large observational study of the determinants of fracture in older men. Contemp Clin Trials 2005;26: 569-585

21. Blackwell T, Ancoli-Israel S, Redline S, Stone $\mathrm{KL}$; Osteoporotic Fractures in Men (MrOS) Study Group. Factors that may influence the classification of sleep-wake by wrist actigraphy: the MrOS Sleep Study. J Clin Sleep Med 2011;7:357367

22. Cole RJ, Kripke DF, Gruen W, Mullaney DJ, Gillin JC. Automatic sleep/wake identification from wrist activity. Sleep 1992;15:461-469

23. Ancoli-Israel S, Clopton P, Klauber MR, Fell R, Mason W. Use of wrist activity for monitoring sleep/wake in demented nursing-home patients. Sleep 1997;20:24-27

24. Marler MR, Gehrman P, Martin JL, AncoliIsrael $\mathrm{S}$. The sigmoidally transformed cosine curve: a mathematical model for circadian rhythms with symmetric non-sinusoidal shapes. Stat Med 2006;25:3893-3904

25. Lee CG, Boyko EJ, Strotmeyer ES, et al.; Osteoporotic Fractures in Men Study Research Group. Association between insulin resistance and lean mass loss and fat mass gain in older men without diabetes mellitus. J Am Geriatr Soc 2011; 59:1217-1224

26. Matthews DR, Hosker JP, Rudenski AS, Naylor BA, Treacher DF, Turner RC. Homeostasis model assessment: insulin resistance and betacell function from fasting plasma glucose and insulin concentrations in man. Diabetologia 1985; 28:412-419

27. Boucher B, Cotterchio $M$, Kreiger N, Nadalin $\mathrm{V}$, Block T, Block $\mathrm{G}$. Validity and reliability of the Block98 food-frequency questionnaire in a sample of Canadian women. Public Health Nutr 2006; 9:84-93

28. Rogers TS, Harrison S, Judd S, et al.; Osteoporotic Fractures in Men (MrOS) Study Research Group. Dietary patterns and longitudinal change in hip bone mineral density among older men. Osteoporos Int 2018;29:1135-1145

29. Washburn RA, Smith KW, Jette AM, Janney CA. The Physical Activity Scale for the Elderly (PASE): development and evaluation. J Clin Epidemiol 1993;46:153-162

30. Schuit AJ, Schouten EG, Westerterp KR, Saris WH. Validity of the Physical Activity Scale for the Elderly (PASE): according to energy expenditure assessed by the doubly labeled water method. J Clin Epidemiol 1997;50:541-546

31. Sheikh JI, Yesavage JA, Brooks JO III, et al. Proposed factor structure of the Geriatric Depression Scale. Int Psychogeriatr 1991;3:23-28 32. Schisterman EF, Cole SR, Platt RW. Overadjustment bias and unnecessary adjustment in epidemiologic studies. Epidemiology 2009;20: 488-495

33. Cole SR, Platt RW, Schisterman EF, et al. Illustrating bias due to conditioning on a collider. Int J Epidemiol 2010;39:417-420

34. Moskvina V, Schmidt KM. On multiple-testing correction in genome-wide association studies. Genet Epidemiol 2008;32:567-573

35. Greenland S, Senn SJ, Rothman KJ, et al. Statistical tests, $P$ values, confidence intervals, and power: a guide to misinterpretations. Eur J Epidemiol 2016;31:337-350

36. Cespedes Feliciano EM, Quante M, Weng J, et al. Actigraphy-derived daily rest-activity patterns and body mass index in community-dwelling adults. Sleep (Basel) 2017;40:zsx168
37. Weir GC, Bonner-Weir S. Five stages of evolving beta-cell dysfunction during progression to diabetes. Diabetes 2004;53(Suppl. 3): S16-S21

38. $\mathrm{Yu} \mathrm{JH}$, Yun $\mathrm{CH}, \mathrm{Ahn} \mathrm{JH}$, et al. Evening chronotype is associated with metabolic disorders and body composition in middle-aged adults. J Clin Endocrinol Metab 2015;100: 1494-1502

39. Knutson KL, von Schantz M. Associations between chronotype, morbidity and mortality in the UK Biobank cohort. Chronobiol Int 2018;35: 1045-1053

40. Gabriel BM, Zierath JR. Circadian rhythms and exercise - re-setting the clock in metabolic disease. Nat Rev Endocrinol 2019;15:197-206 41. Savikj M, Gabriel BM, Alm PS, et al. Afternoon exercise is more efficacious than morning exercise at improving blood glucose levels in individuals with type 2 diabetes: a randomised crossover trial. Diabetologia 2019;62:233-237 42. Ezagouri S, Zwighaft Z, Sobel J, et al. Physiological and molecular dissection of daily variance in exercise capacity. Cell Metab 2019;30: 78-91.e4

43. Stenvers DJ, Scheer FAJL, Schrauwen $P$, la Fleur SE, Kalsbeek A. Circadian clocks and insulin resistance. Nat Rev Endocrinol 2019;15:75-89

44. Marcheva B, Ramsey KM, Buhr ED, et al. Disruption of the clock components CLOCK and BMAL1 leads to hypoinsulinaemia and diabetes. Nature 2010;466:627-631

45. Scheer FA, Hilton MF, Mantzoros CS, Shea SA. Adverse metabolic and cardiovascular consequences of circadian misalignment. Proc Natl Acad Sci U S A 2009;106:4453-4458

46. Qian J, Dalla Man C, Morris CJ, Cobelli C, Scheer FAJL. Differential effects of the circadian system and circadian misalignment on insulin sensitivity and insulin secretion in humans. Diabetes Obes Metab 2018;20:2481-2485

47. Lyytikäinen $P$, Lallukka T, Lahelma E, Rahkonen O. Sleep problems and major weight gain: a follow-up study. Int J Obes 2011;35:109-114 48. Qian J, Morris CJ, Caputo R, Wang W, Garaulet $M$, Scheer FAJL. Sex differences in the circadian misalignment effects on energy regulation. Proc Natl Acad Sci U S A 2019;116: 23806-23812 